\title{
Addendum to the Acknowledgments: Stepwise-Hierarchical Pooled Analysis for Synergistic Interpretation of Meta-analyses Involving Randomized and Observational Studies: Methodology Development
}

In-Soo Shin ${ }^{1}, \mathrm{PhD}$; Chai Hong Rim ${ }^{2}, \mathrm{MD}, \mathrm{PhD}$

${ }^{1}$ Graduate School of Education, Dongguk University, Seoul, Republic of Korea
${ }^{2}$ Department of Radiation Oncology, Ansan Hospital, Korea University, Gyeonggido, Republic of Korea

Corresponding Author:

Chai Hong Rim, MD, PhD

Department of Radiation Oncology

Ansan Hospital

Korea University

Jeokgeumro 123

Danwon $\mathrm{Gu}$

Gyeonggido, 15355

Republic of Korea

Phone: 82314126850

Fax: 82314126851

Email: crusion3@naver.com

\section{Related Article:}

Correction of: https://www.jmir.org/2021/9/e29642

(J Med Internet Res 2021;23(9):e33534) doi: 10.2196/33534

In "Stepwise-Hierarchical Pooled Analysis for Synergistic Interpretation of Meta-analyses Involving Randomized and Observational Studies: Methodology Development" (J Med Internet Res 2021;23(9):e29642), two errors were noted.

In the originally published article, the Acknowledgments section was incorrect. This section originally read as follows:

This study was supported by the National Research Fund of Korea (NRF-2018R1D1A1B07046998). The funders had no role in the study design, data collection and analysis, decision to publish, or preparation of the manuscript.

This has been corrected to:
This study was supported by the National Research Fund of Korea (NRF-2018R1D1A1B07046998 and NRF-2019M2D2A1A01031560). The funders had no role in the study design, data collection and analysis, decision to publish, or preparation of the manuscript.

As well, in the originally published article, the postal code of the Corresponding Author was incorrectly displayed as "13555." This has been corrected to "15355."

The correction will appear in the online version of the paper on the JMIR Publications website on September 20, 2021, together with the publication of this correction notice. Because this was made after submission to PubMed, PubMed Central, and other full-text repositories, the corrected article has also been resubmitted to those repositories.

This is a non-peer-reviewed article. Submitted 11.09.21; accepted 13.09.21; published 20.09.21.

Please cite as:

Shin IS, Rim $C H$

Addendum to the Acknowledgments: Stepwise-Hierarchical Pooled Analysis for Synergistic Interpretation of Meta-analyses Involving

Randomized and Observational Studies: Methodology Development

J Med Internet Res 2021;23(9):e33534

URL: https://www.jmir.org/2021/9/e33534

doi: $10.2196 / 33534$

PMID: 
CIn-Soo Shin, Chai Hong Rim. Originally published in the Journal of Medical Internet Research (https://www.jmir.org), 20.09.2021. This is an open-access article distributed under the terms of the Creative Commons Attribution License (https://creativecommons.org/licenses/by/4.0/), which permits unrestricted use, distribution, and reproduction in any medium, provided the original work, first published in the Journal of Medical Internet Research, is properly cited. The complete bibliographic information, a link to the original publication on https://www.jmir.org/, as well as this copyright and license information must be included. 